\title{
Health and safety
}

- A hazard is anything that may cause harm, such as chemicals, electricity, working from ladders, an open drawer, etc.

- The risk is the chance, high or low, that somebody could be harmed by these and other hazards, together with an indication of how serious the harm could be.

\section{Control of substances hazardous to health}

The 2002 COSHH Regulations require employers to control substances that are hazardous to health. You can prevent or reduce workers' exposure to hazardous substances by:

- Finding out what the health hazards are

- Deciding how to prevent harm to health (risk assessment)

- Providing control measures to reduce harm to health

- Making sure control measures are used

- Keeping all control measures in good working order

- Providing information, instruction and training for employees and others

- Providing monitoring and health surveillance in appropriate cases

- Planning for emergencies.

Every employer or self-employed person is legally required to make an assessment of the health and safety risks arising out of their work. The purpose of the assessment is to identify what needs to be done to control health and safety risks. If a practice employs five or more people, the assessment(s) must be recorded in writing. Failure to adequately control hazards can lead to prosecution under the COSHH Regulations and civil action from injured or ill employees. A hazardous substance may be defined as any substance which can cause adverse health effects or disease.

Hazardous substances include:

- Substances used directly in work activities and classified as dangerous to health under the Registration, Evaluation, Authorisation and Restriction of Chemicals (REACH) legislation 2007 (recognizable by their warning symbols) (e.g. cleaning agents)

- Substances generated during work activities (e.g. waste fumes from anaesthesia equipment)

- Naturally occurring substances (e.g. dust from litter trays, if concentrations in the air exceed levels specified in the COSHH Regulations)

- Biological agents, such as bacteria and other microorganisms

- Other substances that may pose a risk to health, but not covered by the Classification Labelling and Packaging (CLP) Regulations for technical reasons (e.g. medicines). 
To comply with $\mathrm{COSHH}$ Regulations, the following eight steps must be followed:

1. Assess the risks.

2. Decide what precautions are needed.

3. Prevent or adequately control exposure.

4. Ensure that control measures are used and maintained.

5. Monitor the exposure.

6. Carry out appropriate health surveillance.

7. Prepare plans and procedures to deal with accidents, incidents and emergencies.

8. Ensure employees are properly informed, trained and supervised.

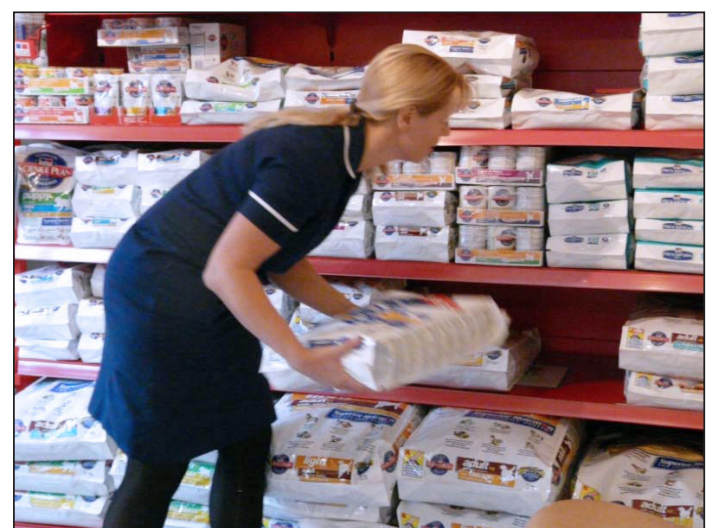

Safety datasheets provide information regarding the substance. They do not constitute the COSHH assessment. The assessment must be task-based, considering the quantity used, the way in which the substances are used, who will be affected and how.

\section{Risk assessment}

A risk assessment involves five steps:

1. Identify the hazards.

2. Decide who might be harmed and how.

3. Evaluate the risks and decide on precautions.

4. Record your significant findings.

5. Review your assessment and update if necessary.

Areas of work in the dispensary requiring risk assessment include:

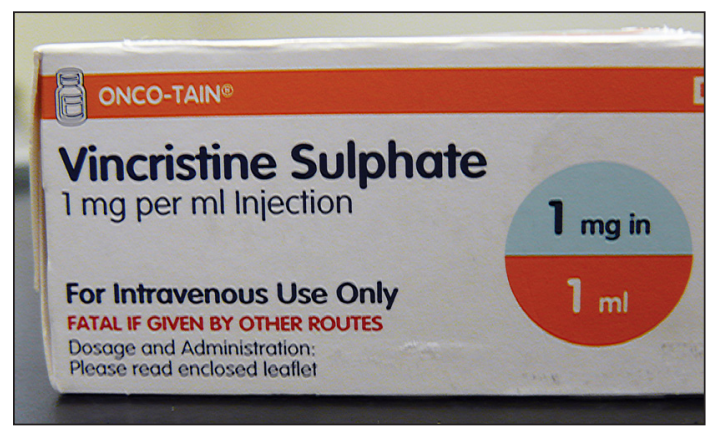

- General medicines handling

- Handling cytotoxic drugs

- Spillage of medicines

- Manual handling: accessing high shelves, moving drug order, etc.

- Trip hazards

- Waste disposal.

The HSE has produced a blank sample risk assessment that may be used either as a template or as a guide to creating a practice-specific risk assessment document. It is available for download from the HSE website (@).

Risk assessments should be carried out for all these tasks and reviewed annually. Standard operating procedures (SOPs) should be written detailing the required control methods, and all staff should be required to sign to acknowledge that the SOPs have been read and understood.

When working with veterinary medicines, there is a wide variation in risk. Many medicines can be classified as low or medium risk but others pose a very serious risk to health.

See also Correct storage, dispensary management and standard operating procedures.

See also Cytotoxic drugs.

\section{Low and medium risk substances}

Risks associated with handling low and medium risk medicines can be adequately controlled by performing assessments by therapeutic group/type/route of administration. For example, the practice can produce standard methods for the control of exposure to:

- Injectable anaesthetics

- Inhalation anaesthetics

- Pour on anthelmintics

- Steroidal compounds

- Vaccines

- Antibiotics

- Disinfectants.

Within these groups, practices must identify specific risks such as penicillin allergy. 


\section{High risk substances}

Specific and detailed assessments along with the resulting control methods should be made for high risk substances such as:

- Cytotoxic drugs

- Tilmicosin (e.g. Micotil)

- Hormones

- Oil-based vaccines

- Glutaraldehyde disinfectants

- Large animal etorphine.

In general, when handling medicines members of staff must:

- Treat all medicinal products as potentially harmful

- Be aware of the hazards associated with medicines and know the results of the COSHH and risk assessments

- Wear disposable gloves when handling any open or loose products

- Be familiar with the practice SOPs for handling medicines and use additional protective clothing and equipment as and when specified

- Inform the health and safety officer if they (or their partner) are or are trying to become pregnant. In the case of pregnancy, be aware of and avoid handling teratogenic drugs (see the BSAVA Small Animal Formulary for a listing) likely to harm the unborn child, or drugs likely to cause miscarriage. In the case of trying to become pregnant, be aware of and avoid mutagenic drugs, which can affect eggs and sperm

- Inform the health and safety officer if they experience any allergies or adverse effects thought to be caused or made worse by the handling of, or exposure to, veterinary medicinal products (VMP)

- Wash their hands after handling medicines, even if disposable gloves have been worn.

\section{Summaries of product characteristics}

To perform risk assessments, employers require information on the safe use of medicines, chemicals and disinfectants.

Manufacturers are no longer required to produce safety datasheets for medicines. Information of the safe use of each medicine can be found in the SPC. The Veterinary Medicines Directorate product information database (@) has a full list of veterinary SPCs for:

- Currently authorized products

- Expired products

- Suspended products

- Registered homeopathic products

- Refused products

- Specified feed additives.

For non-veterinary authorized drugs (e.g. human prescription-only medicines) used under the prescribing cascade, SPCs can be found online (@).

Chemicals and disinfectants are required under REACH regulations 2007 (@) to have a safety datasheet and appropriate warning symbols on the product packaging.

\section{RIDDOR and reporting incidents}

Under the Reporting of Injuries, Diseases and Dangerous Occurrences Regulations 2013 (RIDDOR), the accidental release of any substance that may cause a major injury or damage to health, is classed as a dangerous occurrence and should be reported to the HSE. However, a small spillage of a cytotoxic drug, which is well contained and easily dealt with, is not reportable. Spillage of a large amount, to which people could have been exposed, is reportable.

In addition, there are a number of specific injuries that must be reported:

- Fractures, other than to fingers, thumbs and toes

- Amputations

- Any injury likely to lead to permanent loss of sight or reduction in sight

- Any crush injury to the head or torso causing damage to the brain or internal organs

- Serious burns (including scalding) which

- Covers more than $10 \%$ of the body

- Causes significant damage to the eyes, respiratory system or other vital organs

- Any scalping requiring hospital treatment

- Any loss of consciousness caused by head injury or asphyxia

- Any other injury arising from working in an enclosed space which

- Leads to hypothermia or heat-induced illness

- Requires resuscitation or admittance to hospital for more than 24 hours.

Accidents must be reported, where they result in an employee or self-employed person being away from work, or unable to perform their normal work duties for more than seven consecutive days as the result of their injury. Any accident or injury that prevents an employee or self-employed person from performing work duties for more than three consecutive days must be recorded in an accident book, but is not notifiable. 


\section{How to report}

Online: go to the website (@) and complete the appropriate online report form. The form will then be submitted directly to the RIDDOR database. You will receive a copy for your records.

Telephone: all incidents can be reported online but a telephone service remains for reporting fatal and specified injuries only. Call the Incident Contact Centre on 08453009923 (opening hours Monday to Friday 8.30 am to 5 pm).

\section{Further information}

- For further information on COSHH regulations 2002, see the HSE website (@)

- For further information on risk assessments, see the HSE website (@)

- For further information on REACH, see the HSE website (@)

- For further information on CLP, see the HSE website (

\section{QUESTIONS}

1. Risk assessments that should be carried out for the dispensary include:
a. Spillage of medicines
b. Manual handling
c. Handling of cytotoxic medicines
d. All of the above

2. Specific and detailed COSHH assessments must be performed for which one of the following?
a. Steroids
b. Inhalation anaesthetics
c. Hormones
d. Antibiotics

3. The VMD website does not hold SPCs for which one of the following?
a. Currently authorized veterinary medicines
b. Human medicines
c. Suspended products
d. Registered homeopathic products

4. Accidents or injuries that result in an employee being off work for what length of time must be reported under RIDDOR?
a. 1 day
b. 3 consecutive days
c. 7 consecutive days
d. 10 consecutive days 